\title{
Analysis of 90/150 CA Concatenated using Inverse Symmetric Transition Rule
}

\author{
Un Sook Choi ${ }^{1, \mathrm{a}}$ Sung Jin Cho*2,b, Han Doo Kim ${ }^{3, \mathrm{c}}$, Min Jeong Kwon ${ }^{2, \mathrm{~d}}$, \\ Jin Gyoung Kim ${ }^{2, \mathrm{e}}$, Sung Won Kang ${ }^{2, \mathrm{f}}$ \\ ${ }^{1}$ Department of Information and Communications Engineering, Tongmyong \\ University, 428, Sinseon-ro, Nam-Gu, Busan, 48520, Korea, \\ ${ }^{2}$ Department of Applied Mathematics, Pukyong National University, 45 Yongso-ro, \\ Nam-Gu, Busan, 48513, Korea, \\ ${ }^{3}$ Institute of Basic Sciences and Department of Applied Mathematics, Inje \\ University, Inje-ro, Gimhae-si, Gyeongnam, 50834, Korea,

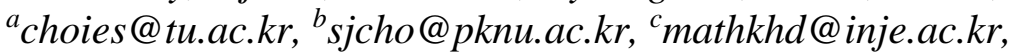

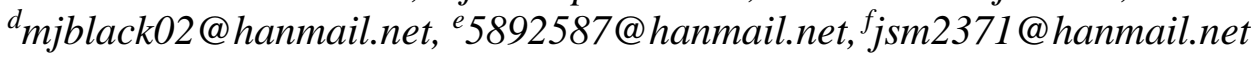

\begin{abstract}
In symmetric cryptosystems, key sequence generation is a very important problem. Various key sequence generation methods have been proposed by many researchers. A shrinking generator consisting of two LFSRs with maximum lengths generates a key sequence in nonlinear manners. Since the characteristic polynomial of the shrunken sequence is of the form $\{f(x)\}^{k}(k \in \mathbb{N})$, it can be modeled as a linear 90/150 Cellular Automata (CA). In this paper, we analyze a linear 90/150 CA using inverse symmetric transition rules of various types. By the proposed method we generate $(2 n+i)$-cell $(i=0,1,2$, $\cdots, n)$ 90/150 CA based on n-cell 90/150 CA. Especially we synthesize 90/150 CA corresponding to $\{f(x)\}^{k}$ using inverse symmetric transition rules. Also, we show that the probabilities of finding $(2 n+i)$-cell $C A \mathbb{C}$ with maximum lengths by the proposed method are greater than the probabilities of finding the $\mathbb{C}$ by an exhaustive search.
\end{abstract}

Keywords: 90/150 Cellular Automata; Characteristic Polynomial; Inverse Symmetric Transition Rule; Shrinking Generator; Key Sequence.

\section{Introduction}

Wolfram in early 1980's suggested a simplified two-state three-neighborhood 1-D CA with cells arranged linearly in one dimension [1]. CA has a simple, regular, modular and cascadable structure with logical neighborhood interconnection. The simple structure of CA with logical interconnections is ideally suited for hardware implementation. For these reasons CA have been used for various applications such as pattern classification ([2], [3]), cryptography $([4] \sim[8])$, and pseudorandom-number generation, etc. ([9] [12]). Cho et al. ([11], [13]) analyzed characteristic polynomials of group CA and non-group CA. Moreover they proposed efficient methods of synthesis of 90/150 maximum length CA [13]. Choi et al. [14] proposed the 90/150 CA synthesis method whose characteristic polynomial has maximum weight. Cho et al. [11] and Sabater et al. [15] proposed classes of cryptographic interleaved sequences generated by linear 90/150 CA obtained by concatenating the basic automaton. The interleaved sequence generator in [11] and [15] has a limitation that characteristic polynomial of the generator is $\{f(x)\}^{2^{a}}(a \in \mathbb{N})$. To

* Corresponding author : Sung Jin Cho; E-mail: sjcho@pknu.ac.kr 
overcome this restriction, we propose the method to model the shrinking generator as $90 / 150 \mathrm{CA}$ whose characteristic polynomial is of the form $\{f(x)\}^{k}(k \in \mathbb{N})$ in this paper. And we analyze 90/150 CA using inverse symmetric transition rule of various types. Also we give a method of generating $(2 n+i)$-cell $(i=0,1,2, \cdots, n) 90 / 150$ CA based on $n$-cell 90/150 CA. Especially we give $(2 n+i)$-cell 90/150 CA $\mathbb{C}$ whose characteristic polynomials are irreducible or primitive. The probabilities of finding $(2 n+i)$-cell $\mathrm{CA} \mathbb{C}$ are greater than the probabilities of finding the $\mathbb{C}$ by an exhaustive search.

\section{Preliminaries}

A CA consists of a number of interconnected cells arranged spatially in a regular manner [1]. In most simple case, a CA cell can exhibit two different states (0 or 1) and the next state of each cell depends on the present states of its three neighborhoods including itself. The state $s_{i}^{t+1}$ of the $i$ th cell at time $(t+1)$ is denoted as

$$
s_{i}^{t+1}=f_{i}\left(s_{i-1}^{t}, s_{i}^{t}, s_{i+1}^{t}\right),
$$

where $s_{i}^{t}$ denotes the state of the $i$ th cell at time $t$ and $f_{i}$ is the next state function called the rule of the CA. If the next state generating logic employs only XOR logic, then it is called a linear rule. And a CA with all the cells having linear rules is called a linear $C A$ [16]. Since a linear CA employs XOR logic only as the next state function, it can be represented as a matrix referred to as the state transition matrix over the finite field $G F(2)$. An $n$-cell CA is characterized by an $n \times n$ state transition matrix. The state transition matrix $T$ is constructed as

$$
T=\left(\begin{array}{cccccc}
d_{1} & a_{1,2} & 0 & \cdots & 0 & 0 \\
a_{2,1} & d_{2} & a_{2,3} & \cdots & 0 & 0 \\
0 & a_{3,2} & d_{3} & \cdots & 0 & 0 \\
\vdots & \vdots & \vdots & \ddots & \vdots & \vdots \\
0 & 0 & 0 & \cdots & a_{n, n-1} & d_{n}
\end{array}\right)
$$

where $a_{i, j}=1$ if the next state of the $i$ th cell depends on the present state of the $j$ th cell, and $a_{i, j}=0$ otherwise. And $a_{i, i}=d_{i}, i=1,2, \cdots, n$. In this paper, a CA is a nullboundary $90 / 150 \mathrm{CA}$ fully specified by which cells use 90 and 150 . The transition rules 90 and 150 are defined as follows:

$$
\begin{aligned}
& \text { Rule 90: } s_{i}^{t+1}=s_{i-1}^{t} \oplus s_{i+1}^{t} \\
& \text { Rule 150: } s_{i}^{t+1}=s_{i-1}^{t} \oplus s_{i}^{t} \oplus s_{i+1}^{t}
\end{aligned}
$$

According to rule 90, the value of a particular cell $i$ is the sum modulo 2 of the values of its two neighboring cells(left and right) on the previous time step $t$. And in rule 150, the value of a particular cell $i$ is the sum modulo 2 of the values of its two neighboring cells and the value of cell $i$ on the previous time step $t$. A natural form for the specification of $90 / 150 \mathrm{CA}$ is an $n$-tuple $\left\langle d_{1}, d_{2}, \cdots, d_{n}\right\rangle$, called the rule vector, where $d_{i}=0$ (resp. 1) if cell $i$ uses rule 90 (resp. 150).

A polynomial is said to be a CA-polynomial if it is the characteristic polynomial of some 90/150 CA. All irreducible polynomials are CA-polynomials [17]. In [17], Cattell et al. proposed a method for the synthesis of one-dimensional 90/150 Linear Hybrid Group CA (LHGCA) for an irreducible polynomial. Cho et al. [13] proposed 
a new efficient method for the synthesis of one-dimensional 90/150 LHGCA for any CA-polynomial as well as irreducible polynomial by using Lanczos tridiagonalization algorithm. This algorithm is efficient and suitable for all practical applications. Cho et al. [11] and Sabater et al. [15] proposed a method of constructing a linear 90/150 CA with characteristic polynomial $\{f(x)\}^{2}$ by concatenating the basic automaton whose characteristic polynomial is $f(x)$, which is irreducible.

\section{Recurrence relation of $90 / 150 \mathrm{CA}$ using inverse symmetric transition rule}

In this section, we provide theoretical information about concatenation of 90/150 CA using inverse symmetric transition rules of various types. The characteristic polynomial $\Delta_{n}$ of an $n$-cell $90 / 150 \mathrm{CA} \mathbb{C}_{n}$ is defined by $\Delta_{n}=\left|T_{n} \oplus x I_{n}\right|$ where $x$ is an indeterminate, $I_{n}$ is the $n \times n$ identity matrix and $T_{n}$ is the state transition matrix of $\mathbb{C}_{n}$. For any $n$-cell 90/150 CA whose state transition matrix is $T_{n}$, the minimal polynomial for $T_{n}$ is the same as the characteristic polynomial for $T_{n}$ [12]. Let $\Delta_{n}$ be the characteristic polynomial of $T_{n}$. Then the following recurrence relation holds:

$$
\Delta_{n}=\left(x+d_{n}\right) \Delta_{n-1}+\Delta_{n-2}
$$

where $\Delta_{1}=x+d_{1}, \Delta_{0}=1$ [17]. Eq. (1) provides an efficient algorithm to compute $\Delta_{n}$ of a given 90/150 CA from its rule vector. We denote the characteristic polynomial of sub-CA consisting of cells $i$ through $j$ by $\Delta_{i, j}$, where $i \leq j$. We simply denote $\Delta_{1, j}$ by $\Delta_{j} . \quad \Delta_{j}$ is said to be a CA-subpolynomial. Let $T_{n}=<a_{1}, a_{2}, \cdots, a_{n}>\left(\right.$ resp. $\left.T_{n}^{*}=<a_{n}, \cdots, a_{2}, a_{1}>\right)$ be the state transition matrix of an $n$ cell $90 / 150 \mathrm{CA}$ and $\Delta_{n}$ (resp. $\Delta_{n}^{*}$ ) be the characteristic polynomial of $T_{n}$ (resp. $T_{n}^{*}$ ). Then $\Delta_{n}^{*}=\Delta_{n} . T_{n}^{*}$ is called the state transition matrix corresponding to $T_{n}$.

Definition 3.1. [18] Let $T_{n}$ be the state transition matrix of an $n$-cell $90 / 150$ CA and let $T_{n}^{*}$ be the state transition matrix corresponding to $T_{n}$. Let $S_{2 n}$ be the following state transition matrix of a $2 n$-cell $90 / 150 \mathrm{CA} \mathbb{C}_{2 n}$. Then the state transition matrix $\left.S_{2 n}=<T_{n}, T_{n}^{*}\right\rangle=<a_{1}, a_{2}, \cdots, a_{n}, a_{n}, \cdots, a_{2}, a_{1}>$ is called the symmetric transition rule of $\mathbb{C}_{2 n}$.

Let $T_{n}=<a_{1}, a_{2}, \cdots, a_{n}>$ be the state transition matrix of an $n$-cell $90 / 150 \mathrm{CA}$ and $T_{\bar{n}}=\left\langle a_{1}, \cdots, a_{n-1}, \overline{a_{n}}\right\rangle$. And let $V_{2 n}$ be the characteristic polynomial of $M_{2 n}=$ $<T_{\bar{n}}, T_{\bar{n}}^{*}>$. Then the following holds:

$$
V_{2 n}=\left(\Delta_{n}+\Delta_{n-1}\right)^{2}=\left(\Delta_{\bar{n}}\right)^{2}
$$

where $\Delta_{\bar{n}}$ is the characteristic polynomial of $T_{\bar{n}}$. Let $D_{1}$ be the characteristic polynomial of the 1-cell 90/150 CA with the rule vector $\langle d\rangle$. Let $M_{2 n+1}=$ $<T_{n}, d, T_{n}^{*}>$ be the state transition matrix of a $(2 n+1)$-cell 90/150 CA $\mathbb{C}_{2 n+1}$. And let $V_{2 n+1}$ be the characteristic polynomial of $M_{2 n+1}$. Then $V_{2 n+1}=D_{1} \Delta_{n}{ }^{2}$ [18]. 
Definition 3.2. [19] Let $T_{n}$ be the state transition matrix of an $n$-cell 90/150 CA. Then $\overline{T_{n}^{*}}=<\overline{a_{n}}, \cdots, \overline{a_{2}}, \overline{a_{1}}>$ is called the inverse symmetric transition rule of $T_{n}$.

If $\Delta_{n}=C_{n}(x)$ is the characteristic polynomial of $T_{n}$ and $\overline{\Delta_{n}^{*}}$ is the characteristic polynomial of $\overline{T_{n}^{*}}$, then $\overline{\Delta_{n}^{*}}=C_{n}(x+1)$. Thus $\overline{\Delta_{n}^{*}}$ is irreducible whenever $\Delta_{n}$ is irreducible. For example, let $T_{6}=<0,0,0,1,1,0>$. Then $\overline{T_{6}^{*}}=<1,0,0,1,1,1>$. Since $\Delta_{6}=C_{6}(x)=x^{6}+x+1$ is irreducible, $\overline{\Delta_{6}^{*}}=(x+1)^{6}+(x+1)+1=x^{6}+x^{4}+x^{2}+x+1$ is also irreducible.

For $\overline{T_{n}^{*}}$, the following recurrence relation holds:

$$
\overline{\Delta_{n}^{*}}=\left(x+\overline{a_{n}}\right) \overline{\Delta_{n-1}^{*}}+\overline{\Delta_{n-2}^{*}}
$$

where $\overline{\Delta_{1}^{*}}=x+\overline{d_{1}}, \overline{\Delta_{0}^{*}}=1$. Now we investigate the properties about concatenation of the 90/150 CA using the inverse symmetric transition rule.

Theorem 3.3. Let $T_{n}=<a_{1}, a_{2}, \cdots, a_{n}>$ be the state transition matrix of an $n$-cell 90/150 CA. Then the characteristic polynomial $V_{2 n}(x)$ of $R_{2 n}=<T_{n}, \overline{T_{n}^{*}}>$ is

$$
V_{2 n}(x)=\Delta_{n} \overline{\Delta_{n}^{*}}+\Delta_{n-1} \overline{\Delta_{n-1}^{*}}
$$

where $\overline{\Delta_{n-1}^{*}}$ is the characteristic polynomial of $\left\langle\overline{a_{n-1}}, \cdots, \overline{a_{2}}, \overline{a_{1}}\right\rangle$.

Proof. Since the $(n+1)$ th row of $R_{2 n}+x I_{2 n}$ is of the form

$$
<0, \cdots, 0,1, \frac{x+\overline{a_{n}}}{(n+1) \mathrm{th}}, 1,0, \cdots, 0>
$$

, by cofactor expansion along the $(n+1)$ th row of $R_{2 n}+x I_{2 n}$ we obtain the following:

$$
\begin{aligned}
V_{2 n}(x) & =\left(x+\overline{a_{n}}\right) \Delta_{n} \overline{\Delta_{n-1}^{*}}+1 \cdot|A|+1 \cdot|B| \\
& =\left(x+\overline{a_{n}}\right) \Delta_{n} \overline{\Delta_{n-1}^{*}}+\Delta_{n-1} \overline{\Delta_{n-1}^{*}}+\Delta_{n} \overline{\Delta_{n-2}^{*}} \\
& =\left\{\left(x+\overline{a_{n}}\right) \overline{\Delta_{n-1}^{*}}+\overline{\Delta_{n-2}^{*}}\right\} \Delta_{n}+\Delta_{n-1} \overline{\Delta_{n-1}^{*}} \\
& =\Delta_{n} \overline{\Delta_{n}^{*}}+\Delta_{n-1} \overline{\Delta_{n-1}^{*}},
\end{aligned}
$$

where $A$ is the submatrix obtained by removing the $(n+1)$ th row and the $n$th column of $R_{2 n}+x I_{2 n}$ and $B$ is the submatrix obtained by removing the $n$th row and the $(n+1)$ th column of $R_{2 n}+x I_{2 n}$.

Since $\Delta_{n}(x+1)=\overline{\Delta_{n}^{*}}(x)$ and $\Delta_{n-1}(x+1)=\overline{\Delta_{n-1}^{*}}(x), V_{2 n}(x+1)=V_{2 n}(x)$ in Theorem 3.3. Let $F_{n}(x)=x^{2^{n}}+x+1$. Then the degree of every irreducible factor of $F_{n}(x)$ over $G F(2)$ divides $2 n$ [16]. Since $F_{n}(x+1)=F_{n}(x)$, every irreducible factor of $F_{n}(x)$ is the one of $V_{2 n}(x)$. 
For example, for $x^{2^{4}}+x+1=\left(x^{8}+x^{6}+x^{5}+x^{4}+x^{3}+x+1\right)\left(x^{8}+x^{6}+x^{5}+x^{3}+1\right)$, 90/150 CA corresponding to $x^{8}+x^{6}+x^{5}+x^{4}+x^{3}+x+1$ is $<00110011>$ and $90 / 150$ CA corresponding to $x^{8}+x^{6}+x^{5}+x^{3}+1$ (primitive) is $<00001111>$.

To model shrinking generator as $90 / 150 \mathrm{CA}$, we need to synthesize the $90 / 150$ CA corresponding to $\{f(x)\}^{k}(k:$ odd). It is enough to get only the first consecutive $n k-1$ bits $a_{1}, a_{2}, \cdots, a_{n k-1}$ for finding $2 n k$-cell 90/150 CA $<a_{1}, a_{2}, \cdots, a_{n k-1}$, $a_{n k}, \overline{a_{n k}}, \overline{a_{n k-1}}, \cdots, \overline{a_{2}}, \overline{a_{1}}>$ corresponding to $\{f(x)\}^{k}$ and $n=\operatorname{deg} f(x)$. The linear complexity of the shrunken sequence generated by $\{f(x)\}^{k}$ is $2^{L_{1}} \cdot L_{2}$ where $2^{L_{1}-1}<k \leq 2^{L_{1}}$ and $L_{2}=2^{2 n}-1$. Table 1 shows 90/150 CA corresponding to $\left(x^{8}+x^{6}+x^{5}+x^{3}+1\right)^{k}(k=3,5,7,9,11,19)$.

Table 1. 90/150 CA corresponding to $\left(x^{8}+x^{6}+x^{5}+x^{3}+1\right)^{k}$

\begin{tabular}{c|c}
\hline$k$ & $90 / 150 \mathrm{CA}$ (given in hexadecimal notation) \\
\hline \hline $\mathbf{3}$ & 37D413 \\
\hline $\mathbf{5}$ & 3C1F2B07C3 \\
\hline $\mathbf{7}$ & 0D18C2B2BCE74F \\
\hline $\mathbf{9}$ & 0DDF790D4D4F61044F \\
\hline $\mathbf{1 1}$ & 0E48CE7903333F618CED8F \\
\hline$\vdots$ & $\vdots$ \\
\hline $\mathbf{1 9}$ & 37D4318BBEDB52860A71AF9EB524822E73D413 \\
\hline
\end{tabular}

Now we extend the transition rules by adding the 1-cell $\mathrm{CA}\langle d\rangle$ or the 2-cell $\mathrm{CA}<d_{1}, d_{2}>$.

Theorem 3.4. Let $T_{n}=<a_{1}, a_{2}, \cdots, a_{n}>$ be the state transition matrix of an $n$-cell 90/150 CA. Then the following hold:

(1) The characteristic polynomial $V_{2 n+1}$ of $M_{2 n+1}=<T_{n}, d \overline{T_{n}^{*}}>$ is

$$
V_{2 n+1}=\Delta_{n} \overline{\Delta_{n+1}^{*} l_{d}}+\Delta_{n-1} \overline{\Delta_{n}^{*}}
$$

where $\overline{\Delta_{n+1}^{*} l_{d}}$ is the characteristic polynomial of rule vector $\left\langle d, \overline{a_{n}}, \cdots, \overline{a_{2}}, \overline{a_{1}}\right\rangle$.

(2) The characteristic polynomial $V_{2 n+2}$ of $M_{2 n+2}=<T_{n}, d_{1}, d_{2}, \overline{T_{n}^{*}}>$ is

$$
V_{2 n+2}=D_{2} \Delta_{n} \overline{\Delta_{n}^{*}}+\left(x+d_{1}\right) \Delta_{n} \overline{\Delta_{n-1}^{*}}+\Delta_{n-1} \overline{\Delta_{n+1}^{*} l_{d 2}}
$$

where $D_{2}$ is the characteristic polynomial of the 2-cell 90/150 CA with rule vector $\left\langle d_{1}, d_{2}\right\rangle$ and $\Delta_{n+1}^{r_{d_{1}}}$ is the characteristic polynomial of rule vector $<a_{1}, a_{2}, \cdots, a_{n}, d_{1}>$, and $\overline{\Delta_{n+1}^{*} l_{d_{2}}}$ is the characteristic polynomial of rule vector $<d_{2}, \overline{a_{n}}, \cdots, \overline{a_{2}}, \overline{a_{1}}>$.

Proof. We only prove for the case (2). Since the $(n+2)$ th row of $M_{2 n+2}+x I_{2 n+2}$ is of the form

$$
<0, \cdots, 0,1, \frac{x+d_{2}}{(n+2) \mathrm{th}}, 1,0, \cdots, 0>
$$


by cofactor expansion along the $(n+2)$ th row of $M_{2 n+2}+x I_{2 n+2}$ we obtain the following:

$$
\begin{aligned}
V_{2 n+2}= & \Delta_{n+1}^{r_{d_{1}}}\left(x+d_{2}\right) \overline{\Delta_{n}^{*}}+1 \cdot|A|+1 \cdot|B| \\
= & \Delta_{n+1}^{r_{d_{1}}}\left(x+d_{2}\right) \overline{\Delta_{n}^{*}}+\Delta_{n} \overline{\Delta_{n}^{*}}+\Delta_{n+1}^{r_{d_{1}}} \overline{\Delta_{n-1}^{*}} \\
= & \left(x+d_{2}\right)\left\{\left(x+d_{1}\right) \Delta_{n}+\Delta_{n-1}\right\} \overline{\Delta_{n}^{*}}+\Delta_{n} \overline{\Delta_{n}^{*}}+\left\{\left(x+d_{1}\right) \Delta_{n}+\Delta_{n-1}\right\} \overline{\Delta_{n-1}^{*}} \\
= & \left(x+d_{1}\right)\left(x+d_{2}\right) \Delta_{n} \overline{\Delta_{n}^{*}}+\left(x+d_{2}\right) \Delta_{n-1} \overline{\Delta_{n}^{*}}+\Delta_{n} \overline{\Delta_{n}^{*}}+\left(x+d_{1}\right) \Delta_{n} \overline{\Delta_{n-1}^{*}} \\
& +\Delta_{n-1} \overline{\Delta_{n-1}^{*}} \\
= & D_{2} \Delta_{n} \overline{\Delta_{n}^{*}}+\left(x+d_{1}\right) \Delta_{n} \overline{\Delta_{n-1}^{*}}+\left(x+d_{2}\right) \Delta_{n-1} \overline{\Delta_{n}^{*}}+\Delta_{n-1} \overline{\Delta_{n-1}^{*}} \\
= & D_{2} \Delta_{n} \overline{\Delta_{n}^{*}}+\left(x+d_{1}\right) \Delta_{n} \overline{\Delta_{n-1}^{*}}+\Delta_{n-1}\left\{\left(x+d_{2}\right) \overline{\Delta_{n}^{*}}+\overline{\Delta_{n-1}^{*}}\right\} \\
= & D_{2} \Delta_{n} \overline{\Delta_{n}^{*}}+\left(x+d_{1}\right) \Delta_{n} \overline{\Delta_{n-1}^{*}}+\Delta_{n-1} \overline{\Delta_{n+1}^{*} l_{d-2}}
\end{aligned}
$$

where $A$ is the submatrix obtained by removing the $(n+2)$ th row and the $(n+1)$ th column of $M_{2 n+2}+x I_{2 n+2}$ and $B$ is the submatrix obtained by removing the $(n+2)$ th row and the $(n+3)$ th column of $M_{2 n+2}+x I_{2 n+2}$, and $\Delta_{n+1}^{r_{\alpha_{1}}}$ denotes the characteristic polynomial of rule vector $\left\langle a_{1}, a_{2}, \cdots, a_{n}, d_{1}\right\rangle$, and $\overline{\Delta_{n+1}^{* l_{d_{2}}}}$ denotes the characteristic

\begin{tabular}{|c|c|}
\hline$T_{10}=<a_{1}, \cdots, a_{10}>$ & Characteristic polynomial $V_{21}$ of $\left\langle T_{10}, 0, \overline{T_{10}{ }^{*}}\right\rangle$ \\
\hline$\langle\mathbf{0 , 0 , 0 , 0 , 0 , 0 , 0 , 0 , 0 , 1 \rangle}$ & $21,19,18,16,15,13,12,7,6,4,0$ (irreducible) \\
\hline$\langle 0,0,0,0,0,0,0,1,1,1\rangle$ & $21,19,18,16,13,9,7,5,2,1,0$ (primitive) \\
\hline$\langle\mathbf{0 , 0 , 0 , 0 , 0 , 0 , 1 , 0 , 0 , 0 \rangle}$ & $21,19,18,17,16,14,13,3,0$ (primitive) \\
\hline$\langle\mathbf{0 , 0 , 0 , 0 , 0 , 1 , 1 , 0 , 0 , 1 \rangle}$ & $21,19,18,16,15,12,10,9,6,4,3,1,0$ (primitive) \\
\hline$\langle\mathbf{0 , 0 , 0 , 0 , 1 , 0 , 0 , 0 , 0 , 1 \rangle}$ & $21,19,18,16,15,13,12,9,5,2,0$ (primitive) \\
\hline$\langle\mathbf{0 , 0 , 0 , 0 , 1 , 0 , 0 , 1 , 0 , 0 \rangle}$ & $21,19,18,17,16,14,11,10,8,3,2,1,0$ (primitive) \\
\hline$\langle\mathbf{0 , 0 , 0 , 0 , 1 , 0 , 0 , 1 , 0 , 1 \rangle}$ & $21,19,18,16,15,13,12,9,2,1,0$ (primitive) \\
\hline$\langle\mathbf{0 , 0 , 0 , 0 , 1 , 0 , 1 , 0 , 1 , 1 \rangle}\rangle$ & $21,19,18,16,11,10,9,8,7,1,0$ (primitive) \\
\hline
\end{tabular}
polynomial of rule vector $<d_{2}, \overline{a_{n}}, \cdots, \overline{a_{2}}, \overline{a_{1}}>$.

In Theorem 3.4(2), if $d_{2}=\bar{d}_{1}$, then

Otherwise,

$$
V_{2 n+2}=\Delta_{n+1}^{r_{d_{1}}} \overline{\Delta_{n+1}^{*} r_{d_{1}}}+\Delta_{n} \overline{\Delta_{n}^{*}}
$$

$$
\begin{aligned}
V_{2 n+2} & =\left(x+d_{1}\right)^{2} \Delta_{n} \overline{\Delta_{n}^{*}}+\Delta_{n} \overline{\Delta_{n}^{*}}+\left(x+d_{1}\right) \Delta_{n} \overline{\Delta_{n-1}^{*}}+\Delta_{n-1}\left\{\left(x+d_{1}\right) \overline{\Delta_{n}^{*}}+\overline{\Delta_{n-1}^{*}}\right\} \\
& =\left(x+\overline{d_{1}}\right)^{2} \Delta_{n} \overline{\Delta_{n}^{*}}+\left(x+d_{1}\right)\left(\Delta_{n} \overline{\Delta_{n-1}^{*}}+\overline{\Delta_{n}^{*}} \Delta_{n-1}\right)+\Delta_{n-1} \overline{\Delta_{n-1}^{*}}
\end{aligned}
$$

Table 2 shows the characteristic polynomial $V_{21}$ for the case $d=0$.

Table 2. Characteristic Polynomial $V_{21}$ for the Case $d=0$ 


\begin{tabular}{l|l}
\hline$\langle\mathbf{0 , 0}, \mathbf{0}, \mathbf{0}, \mathbf{1}, \mathbf{1}, \mathbf{0}, \mathbf{1}, \mathbf{0}, \mathbf{0}>$ & $21,19,18,17,16,14,11,10,8,6,4,1,0$ (primitive) \\
\hline$\langle\mathbf{0 , 0}, \mathbf{0}, \mathbf{0}, \mathbf{1}, \mathbf{1}, \mathbf{1}, \mathbf{0}, \mathbf{1}, \mathbf{1}>$ & $21,19,18,16,11,10,9,8,7,6,4,3,2,1,0$ (primitive) \\
\hline
\end{tabular}

Table 3 shows the characteristic polynomial $V_{21}$ for the case $d=1$.

Table 3. Characteristic Polynomial $V_{21}$ for the Case $d=1$

\begin{tabular}{|c|c|}
\hline$T_{10}=<a_{1}, \cdots, a_{10}>$ & Characteristic polynomial $V_{21}$ of $\left\langle T_{10}, 1, \overline{T_{10}^{*}}\right\rangle$ \\
\hline$\langle\mathbf{0 , 0 , 0 , 0 , 0 , 0 , 0 , 0 , 0 , 1 \rangle}$ & $21,20,19,15,14,12,11,10,8,6,4,3,2,1,0$ (primitive) \\
\hline$\langle 0,0,0,0,0,0,0,1,1,1\rangle$ & $21,20,19,13,12,7,6,1,0$ (primitive) \\
\hline$\langle\mathbf{0 , 0 , 0 , 0 , 0 , 0 , 1 , 0 , 0 , 0 \rangle}$ & $21,20,19,17,16,14,13,10,9,6,4,1,0$ (primitive) \\
\hline$\langle 0,0,0,0,0,1,1,0,0,1\rangle$ & $21,20,19,15,14,13,11,7,4,3,0$ (irreducible) \\
\hline$\langle\mathbf{0 , 0 , 0 , 0 , 1 , 0 , 0 , 0 , 0 , 1 \rangle}$ & $21,20,19,15,14,12,11,10,9,7,6,4,0$ (primitive) \\
\hline$\langle\mathbf{0 , 0 , 0 , 0 , 1 , 0 , 0 , 1 , 0 , 0 \rangle}$ & $21,20,19,17,16,14,12,11,10,9,6,5,3,2,0$ (primitive) \\
\hline$\langle 0,0,0,0,1,0,0,1,0,1\rangle$ & $21,20,19,15,14,12,11,10,9,7,6,5,0$ (primitive) \\
\hline$\langle 0,0,0,0,1,0,1,0,1,1\rangle$ & $21,20,19,11,7,6,3,2,0$ (primitive) \\
\hline$\langle 0,0,0,0,1,1,0,1,0,0\rangle$ & $21,20,19,17,16,14,12,11,10,9,5,1,0$ (primitive) \\
\hline$\langle 0,0,0,0,1,1,1,0,1,1\rangle$ & $21,20,19,11,7,1,0$ (primitive) \\
\hline
\end{tabular}

Table 4 shows the characteristic polynomial $V_{22}$ for the case $\left(d_{1}, d_{2}\right)=(0,0)$.

Table 4. Characteristic Polynomial $V_{22}$ for the Case $\left(d_{1}, d_{2}\right)=(0,0)$

\begin{tabular}{|c|c|}
\hline$T_{10}=<a_{1}, \cdots, a_{10}>$ & Characteristic polynomial $V_{22}$ of $\left.<T_{10}, 0,0, \overline{T_{10}{ }^{*}}\right\rangle$ \\
\hline$\langle\mathbf{0 , 0 , 0 , 0 , 0 , 0 , 0 , 0 , 0 , 0 \rangle}$ & $22,19,18,15,14,12,7,6,3,2,0$ (primitive) \\
\hline$\langle\mathbf{0}, \mathbf{0}, \mathbf{0}, \mathbf{0}, \mathbf{0}, \mathbf{0}, \mathbf{0}, \mathbf{0}, \mathbf{1}, \mathbf{1}\rangle$ & $22,19,16,14,13,9,6,2,0$ (primitive) \\
\hline$\langle\mathbf{0}, \mathbf{0}, \mathbf{0}, \mathbf{1}, \mathbf{0}, \mathbf{1}, \mathbf{1}, \mathbf{0}, \mathbf{1}, \mathbf{1}\rangle$ & $22,19,16,13,8,5,4,1,0$ (primitive) \\
\hline$\langle\mathbf{0 , 0}, \mathbf{0}, \mathbf{1}, \mathbf{1}, \mathbf{0}, \mathbf{0}, \mathbf{1}, \mathbf{0}, \mathbf{0}\rangle$ & $22,19,18,15,14,11,8,5,4,1,0$ (irreducible) \\
\hline$\langle\mathbf{0 , 0 , 0 , 1 , 1 , 0 , 1 , 0 , 0 , 0 \rangle}$ & $22,19,18,15,14,12,11,10,9,8,7,6,5,4,3,1,0$ (primitive) \\
\hline$\langle\mathbf{0 , 0 , 0 , 1 , 1 , 1 , 0 , 0 , 0 , 1 \rangle}$ & $22,19,14,12,11,9,8,7,6,5,4,3,0$ (primitive) \\
\hline$\langle\mathbf{0 , 0 , 0 , 1 , 1 , 1 , 0 , 0 , 1 , 0 \rangle}$ & $22,19,18,16,15,14,13,11,7,6,0$ (irreducible) \\
\hline$\langle 0,0,0,1,1,1,0,1,1,0\rangle$ & $22,19,18,16,15,13,12,9,7,6,4,3,0$ (primitive) \\
\hline$\langle\mathbf{0 , 0 , 1 , 0 , 0 , 0 , 0 , 0 , 0 , 1 \rangle}$ & $22,19,14,12,11,9,7,4,0$ (primitive) \\
\hline$\langle\mathbf{0 , 0}, \mathbf{1}, \mathbf{0}, \mathbf{0}, \mathbf{0}, \mathbf{0}, \mathbf{0}, \mathbf{1 , 0}\rangle$ & $22,19,18,16,15,14,13,11,10,3,0$ (irreducible) \\
\hline
\end{tabular}

Table 5 shows the characteristic polynomial $V_{22}$ for the case $\left(d_{1}, d_{2}\right)=(1,1)$.

Table 5. Characteristic Polynomial $V_{22}$ for the Case $\left(d_{1}, d_{2}\right)=(1,1)$

\begin{tabular}{l|l}
\hline$T_{10}=<a_{1}, \cdots, a_{10}>$ & Characteristic polynomial $V_{22}$ of $<T_{10}, 1,1, \overline{T_{10}{ }^{*}}>$ \\
\hline \hline$<\mathbf{0 , 0 , 0 , 0 , 0 , 0 , 0 , 0 , 0 , 0 >}$ & $22,20,19,18,17,16,15,13,12,11,9,8,6,2,0$ (primitive) \\
\hline$<\mathbf{0 , 0 , 0 , 0 , 0 , 0 , 0 , 0 , 1 , 1 >}$ & $22,20,19,17,16,14,13,10,8,6,5,3,2,1,0$ (primitive) \\
\hline$<\mathbf{0 , 0 , 0 , 1 , 0 , 1 , 1 , 0 , 1 , 1 >}$ & $22,20,19,17,16,13,12,9,6,3,0$ (primitive) \\
\hline$<\mathbf{0 , 0 , 0 , 1 , 1 , 0 , 0 , 1 , 0 , 0 >}$ & $22,20,19,18,17,16,15,13,10,7,6,4,3,1,0$ (irreducible) \\
\hline$<\mathbf{0 , 0 , 0 , 1 , 1 , 0 , 1 , 0 , 0 , 0 >}$ & $22,20,19,18,17,16,15,13,12,9,8,6,5,3,0$ (primitive) \\
\hline$<\mathbf{0 , 0 , 0 , 1 , 1 , 1 , 0 , 0 , 0 , 1 >}$ & $22,20,19,17,14,11,8,7,4,2,0$ (primitive) \\
\hline$<\mathbf{0 , 0}, \mathbf{0}, \mathbf{1}, \mathbf{1}, \mathbf{1}, \mathbf{0 , 0}, \mathbf{1}, \mathbf{0}>$ & $22,20,19,18,17,15,12,10,9,6,5,1,0$ (primitive) \\
\hline
\end{tabular}




\begin{tabular}{l|l}
\hline$\langle\mathbf{0 , 0 , 0 , 1 , 1 , 1 , 0 , 1 , 1 , 0 >}$ & $22,20,19,18,17,15,14,12,11,10,9,5,4,2,0$ (irreducible) \\
\hline$\langle\mathbf{0 , 0 , 1 , 0 , 0 , 0 , 0 , 0 , 0 , 1}>$ & $22,20,19,17,14,11,7,6,5,4,3,2,0$ (irreducible) \\
\hline$\langle\mathbf{0 , 0 , 1 , 0 , 0 , 0 , 0 , 0 , 1 , 0 >}$ & $22,20,19,18,17,15,12,9,8,7,6,1,0$ (primitive) \\
\hline
\end{tabular}

In Theorem 3.4, $<d_{1}, d_{2}>$ can be extended to $D_{k}=<d_{1}, d_{2}, \cdots, d_{k}>(k \leq n)$.

Remark. If the characteristic polynomial $C_{2 n+k}(x)$ of a $(2 n+k)$-cell 90/150 CA $<T_{n}, D_{k}, \overline{T_{n}^{*}}>=<a_{1}, \cdots, a_{n}, d_{1}, \cdots, d_{k}, \overline{a_{n}}, \cdots, \overline{a_{1}}>$ is irreducible, then the characteristic polynomial of $<T_{n}, \overline{D_{k}^{*}}, \overline{T_{n}^{*}}>$ is irreducible.

We can obtain $(2 n+i)$-cell $(i=0,1,2, \cdots, n) 90 / 150 \mathrm{CA}$ using $T_{n}$ and $D_{k}(k \leq n)$ by Theorem 3.3, Theorem 3.4 and Remark.

By Remark, we obtain Tables 6 and 7. Table 6 provides a list of $n$-cell $(8 \leq n \leq 12)$ $90 / 150 \mathrm{CA} \mathbb{C}$ and irreducible polynomials corresponding to $\mathbb{C}$, which are obtained by the proposed method using 4-cell $90 / 150 \mathrm{CA}$ and $D_{k}(k=1,2,3,4)$. Also Table 7 shows the comparison of frequency rates of irreducible polynomials by exhaustive method and the proposed method. It is shown that the probability of finding the irreducible polynomial by the proposed method is greater than the probability of finding the irreducible polynomials by the exhaustive method.

Table 6. 90/150 CA $\mathbb{C}$ and Irreducible Polynomials corresponding to $\mathbb{C}$

\begin{tabular}{|c|c|c|c|}
\hline Degree & $T_{n}$ & $D_{k}$ & $\begin{array}{c}\text { Characteristic } \\
\text { Polynomial }\end{array}$ \\
\hline 8 & $\begin{array}{l}<0,0,0,0> \\
<1,1,0,0> \\
<1,1,1,1>\end{array}$ & $\begin{array}{l}- \\
- \\
-\end{array}$ & $\begin{array}{l}8,6,5,3,0 \\
8,6,5,4,3,1,0 \\
8,6,5,3,0\end{array}$ \\
\hline 9 & $\begin{array}{l}<0,0,0,1> \\
<0,1,1,1> \\
<1,0,0,0> \\
<1,1,1,0> \\
<0,0,0,1> \\
<0,1,1,1> \\
<1,0,0,0> \\
<1,1,1,0>\end{array}$ & $\begin{array}{l}<0> \\
<0> \\
<0> \\
<0> \\
<1> \\
<1> \\
<1> \\
<1>\end{array}$ & $\begin{array}{l}9,6,5,3,2,1,0 \\
9,6,5,3,0 \\
9,6,5,3,0 \\
9,6,5,3,2,1,0 \\
9,8,6,5,3,2,0 \\
9,8,6,5,3,1,0 \\
9,8,6,5,3,1,0 \\
9,8,6,5,3,2,0\end{array}$ \\
\hline 10 & $\begin{array}{l}<0,0,0,0> \\
<1,1,1,1> \\
<0,1,0,1> \\
<1,0,1,1> \\
<1,1,0,0> \\
<0,0,1,1> \\
<0,1,0,0> \\
<1,0,1,0> \\
<0,0,0,0> \\
<1,1,1,1>\end{array}$ & $\begin{array}{l}<0,0> \\
<0,0> \\
<0,1> \\
<0,1> \\
<0,1> \\
<1,0> \\
<1,0> \\
<1,0> \\
<1,1> \\
<1,1>\end{array}$ & $\begin{array}{l}10,8,7,6,5,4,3,1,0 \\
10,8,7,6,5,4,3,1,0 \\
10,9,8,6,5,1,0 \\
10,9,8,3,2,1,0 \\
10,9,8,4,3,2,0 \\
10,9,8,4,3,2,0 \\
10,9,8,3,2,1,0 \\
10,9,8,6,5,1,0 \\
10,7,0 \\
10,7,0\end{array}$ \\
\hline 11 & $\begin{array}{l}<0,1,1,1> \\
<1,0,0,0> \\
<0,0,1,0> \\
<1,1,0,1> \\
<0,0,0,0> \\
<1,1,1,1> \\
<0,0,1,0> \\
<1,1,0,1> \\
<0,0,1,0> \\
<1,1,0,1> \\
<0,0,0,0> \\
<1,1,1,1>\end{array}$ & $\begin{array}{l}<0,0,0> \\
<0,0,0> \\
<0,0,1> \\
<0,0,1> \\
<0,1,0> \\
<0,1,0> \\
<0,1,1> \\
<0,1,1> \\
<1,0,0> \\
<1,0,0> \\
<1,0,1> \\
<1,0,1>\end{array}$ & $\begin{array}{l}11,8,5,4,2,1,0 \\
11,8,5,4,2,1,0 \\
11,10,8,7,5,4,3,1,0 \\
11,10,8,6,5,1,0 \\
11,10,8,6,4,2,0 \\
11,10,8,6,4,2,0 \\
11,9,8,7,6,4,3,1,0 \\
11,9,8,6,5,3,2,1,0 \\
11,10,8,6,5,1,0 \\
11,10,8,7,5,4,3,1,0 \\
11,9,8,6,3,1,0 \\
11,9,8,6,3,1,0\end{array}$ \\
\hline
\end{tabular}




\begin{tabular}{|c|c|c|c|}
\hline & $\begin{array}{l}<0,0,1,0> \\
<1,1,0,1> \\
<0,1,1,1> \\
<1,0,0,0>\end{array}$ & $\begin{array}{l}<1,1,0> \\
<1,1,0> \\
<1,1,1> \\
<1,1,1>\end{array}$ & $\begin{array}{l}11,9,8,6,5,3,2,1,0 \\
11,9,8,7,6,4,3,1,0 \\
11,10,9,5,3,1,0 \\
11,10,9,5,3,1,0\end{array}$ \\
\hline 12 & $\begin{array}{l}<0,1,0,0> \\
<0,1,0,1> \\
<0,1,1,1> \\
<1,0,0,0> \\
<1,0,1,0> \\
<1,0,1,1> \\
<0,0,0,0> \\
<0,1,0,1> \\
<1,0,1,0> \\
<1,0,1,1> \\
<0,0,0,1> \\
<0,0,1,0> \\
<0,1,0,0> \\
<0,1,0,1> \\
<1,0,1,0> \\
<0,1,0,1> \\
<0,1,1,1> \\
<1,0,1,1> \\
<0,0,0,0> \\
<1,1,1,1> \\
<0,1,0,0> \\
<1,0,0,0> \\
<1,0,1,0> \\
<0,1,0,1> \\
<1,0,1,0> \\
<1,0,1,1> \\
<1,1,0,1> \\
<1,1,1,0> \\
<0,1,0,0> \\
<0,1,0,1> \\
<1,0,1,0> \\
<1,1,1,1> \\
<0,1,0,0> \\
<0,1,0,1> \\
<0,1,1,1> \\
<1,0,0,0> \\
<1,0,1,0> \\
<1,0,1,1>\end{array}$ & $\begin{array}{l}<0,0,0,0> \\
<0,0,0,0> \\
<0,0,0,0> \\
<0,0,0,0> \\
<0,0,0,0> \\
<0,0,0,0> \\
<0,0,0,1> \\
<0,0,1,0> \\
<0,0,1,0> \\
<0,0,1,0> \\
<0,0,1,1> \\
<0,0,1,1> \\
<0,1,0,0> \\
<0,1,0,0> \\
<0,1,0,0> \\
<0,1,0,1> \\
<0,1,0,1> \\
<0,1,0,1> \\
<0,1,1,1> \\
<1,0,0,0> \\
<1,0,1,0> \\
<1,0,1,0> \\
<1,0,1,0> \\
<1,0,1,1> \\
<1,0,1,1> \\
<1,0,1,1> \\
<1,1,0,0> \\
<1,1,0,0> \\
<1,1,0,1> \\
<1,1,0,1> \\
<1,1,0,1> \\
<1,1,1,0> \\
<1,1,1,1> \\
<1,1,1,1> \\
<1,1,1,1> \\
<1,1,1,1> \\
<1,1,1,1> \\
<1,1,1,1>\end{array}$ & $\begin{array}{l}12,10,9,7,6,5,4,1,0 \\
12,10,9,8,7,3,0 \\
12,10,9,8,7,6,0 \\
12,10,9,8,7,6,0 \\
12,10,9,8,7,3,0 \\
12,10,9,7,6,5,4,1,0 \\
12,11,10,2,0 \\
12,11,10,6,3,2,0 \\
12,11,10,5,2,1,0 \\
12,11,10,8,7,4,0 \\
12,9,8,5,4,1,0 \\
12,9,8,5,0 \\
12,11,10,8,7,4,0 \\
12,11,10,5,2,1,0 \\
12,11,10,6,3,2,0 \\
12,9,5,4,2,1,0 \\
12,9,5,2,0 \\
12,9,8,6,3,2,0 \\
12,11,9,8,4,3,2,1,0 \\
12,11,10,2,0 \\
12,9,8,6,3,2,0 \\
12,9,5,2,0 \\
12,9,5,4,2,1,0 \\
12,11,9,8,6,2,0 \\
12,11,9,8,5,3,2,1,0 \\
12,11,9,7,6,5,4,2,0 \\
12,9,8,5,0 \\
12,9,8,5,4,1,0 \\
12,11,9,7,6,5,4,2,0 \\
12,11,9,8,5,3,2,1,0 \\
12,11,9,8,6,2,0 \\
12,11,9,8,4,3,2,1,0 \\
12,10,9,8,7,4,3,2,0 \\
12,10,9,7,6,5,2,1,0 \\
12,10,9,7,5,4,3,2,0 \\
12,10,9,7,5,4,3,2,0 \\
12,10,9,7,6,5,2,1,0 \\
12,10,9,8,7,4,3,2,0\end{array}$ \\
\hline
\end{tabular}

Table 7. Comparison of Frequency Rates of Irreducible Polynomials by Exhaustive Method and the proposed Method

\begin{tabular}{c|c|c}
\hline Degree & $\begin{array}{c}\text { Frequency Rate of Irreducible } \\
\text { Polynomials by Exhaustive } \\
\text { Method }\end{array}$ & $\begin{array}{c}\text { Frequency Rate of Irreducible } \\
\text { Polynomials by the Proposed } \\
\text { Method }\end{array}$ \\
\hline \hline $\mathbf{8}$ & $23.44 \%$ & $37.50 \%$ \\
\hline $\mathbf{9}$ & $21.88 \%$ & $50.00 \%$ \\
\hline $\mathbf{1 0}$ & $19.34 \%$ & $31.25 \%$ \\
\hline $\mathbf{1 1}$ & $18.16 \%$ & $25.00 \%$ \\
\hline $\mathbf{1 2}$ & $16.36 \%$ & $29.69 \%$ \\
\hline
\end{tabular}

\section{Conclusion}

In this paper, we analysed the characteristic polynomials of 90/150 CA using inverse symmetric transition rule of various types. By the proposed method, we synthesized $(2 n+i)$-cell $(i=0,1,2, \cdots, n) \quad 90 / 150$ CA using $T_{n}$ and $D_{k}(k \leq n)$. 
Especially, we modelled the shrinking generator as 90/150 CA whose characteristic polynomial is $\{f(x)\}^{k}(k \leq n)$ using inverse symmetric transition rules. Also, we showed that the probabilities of finding $(2 n+i)$-cell CA $\mathbb{C}$ with the maximum length by the proposed method are greater than the probabilities of finding $\mathbb{C}$ by an exhaustive search.

\section{Acknowledgments}

This paper is a revised and expanded version of a paper entitled [Synthesis of CA using Linear Rule Blocks] presented at [Proceedings, International Conferences NGCIT 2017, Ho Chi Minh, Vietnam, August 16-18, 2017].

\section{References}

[1] S. Wolfram, "Statistical mechanics of cellular automata", Rev. Modern Physics, vol. 55, no. 3, (1983), pp. 601-644.

[2] K. Bhattacharjee, D. Paul and S. Das, "Pseudorandom pattern generation using 3-state cellular automata", ACRI 2016, LNCS, vol. 9863, (2016), pp. 3-13.

[3] A. Piwonska, F. Seredynski and M. Szaban, "Learning cellular automata rules for binary classification problem", J. Supercomput., vol. 63, (2013), pp. 800-815.

[4] H.B. Adrian, N.M. Mariko, P.M. Hector, A.R. Bucio and M.P. Ramirez-Tachiquin, "A parallel authenticated encryption sharing scheme based on cellular automata", Proceedings of the World Congress on Engineering and Computer Science 2014, vol. 1, (2014), pp. 62-67.

[5] B.R. Gangadari and S.R. Ahamed, "Design of cryptographically secure aes like s-box using secondorder reversible cellular automata for wireless body area network applications", Healthcare Technology Letters, vol. 3, (2016), pp. 177-183.

[6] M. Kumar, S. Kumar, R. Budhiraja, M.K. Das and S. Singh, "Interwining logistic map and cellular automata based color image encryption model.2016", International Conference on Computational Techniques in Information and Communication Technologies (ICCTICT), (2016), pp. 618-623.

[7] S. Roy, S. Nandi, J. Dansana and P.K. Pattnaik, "Application of cellular automata in symmetric key cryptography", IEEE International Conference on Communication and Signal Processing, vol. 114, (2014), pp. 572-576.

[8] S. Singh and R. Bhardwaj, "Image forgery detection using or method based on one dimensional cellular automata", 2016 Ninth International Conference on Contemporary Computing, vol. 3, (2016), pp. 1-5.

[9] K. Bhattacharjee and S. Das, "Reversibility of d-state finite cellular automata", J. Cell. Autom., vol. 11, (2016), pp. 213-245.

[10] S.J. Cho, U.S. Choi, Y.H. Hwang, Y.S. Pyo, H.D. Kim, K.S. Kim and S.H. Heo, "Computing phase shifts of maximum-length 90/150 cellular automata sequences", ACRI 2004, LNCS, vol. 3305, (2004), pp. 31-39.

[11] S.J. Cho, U.S. Choi, H.D. Kim, Y.H. Hwang and J.G. Kim, "Analysis of 90/150 two predecessor nongroup cellular automata”, ACRI 2008, LNCS, vol. 5191, (2008), pp. 128-135.

[12] M. Serra, T. Slater, J.C. Muzio and D.M. Miller, "The analysis of one dimensional linear cellular automata and their aliasing properties", IEEE Trans. Comput-Aided Design Integr. Circuits Syst., vol. 9, no. $7,(\mathbf{1 9 9 0})$, pp. 767-778.

[13] S.J. Cho, U.S. Choi, H.D. Kim, Y.H. Hwang, J.G. Kim and S.H. Heo, "New synthesis of onedimensional 90/150 linear hybrid group cellular automata", IEEE Trans. Comput-Aided Design Integr. Circuits Syst., vol. 26, no. 9, (2007), pp. 1720-1724.

[14] U.S. Choi, S.J. Cho, H.D. Kim and J.G. Kim, "90/150 CA corresponding to polynomial of maximum weight", To appear in J. Cell. Autom.

[15] A.F. Sabater and P.C. Gil, "Synthesis of cryptographic interleaved sequences by means of linear cellular automata", Applied Mathematics Letters, vol. 22, (2009), pp. 1518-1524.

[16] P.P. Chaudhuri, D.R. Chowdhury, S. Nandi and S. Chatterjee, "Additive cellular automata, theory and applications", IEEE Computer Society Press, Los Alamitos, California, vol. 1, (1997).

[17] K. Cattell and J.C. Muzio, "Synthesis of one-dimensional linear hybrid cellular automata", IEEE Trans. Comput-Aided Design Integr. Circuits Syst., vol. 19, no. 2, (1996), pp. 325-335.

[18] U.S. Choi, S.J. Cho and G.T. Kong, "Analysis of characteristic polynomial of cellular automata with symmetrical transition rules", Proceedings of the Jangjeon Mathematical Society, Busan, Korea, (2015), November 20-21. 
[19] S.W. Kang, U.S. Choi, S.J. Cho, H.D. Kim, M.J. Kwon and J.G. Kim, "Synthesis of CA using Linear Rule Blocks", Proceedings, International Conferences NGCIT 2017, Ho Chi Minh, Vietnam, (2017), August 16-18.

\section{Authors}

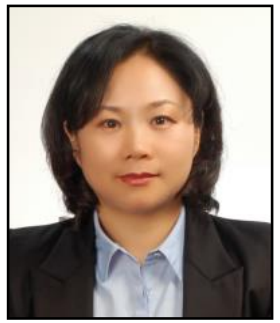

Un Sook Choi, she received the M.S. degree and the Ph.D. degree at Pukyong National University. She is currently a professor at Tongmyong University since 2006. Her research interests include crytography, cellular automata and its applications.

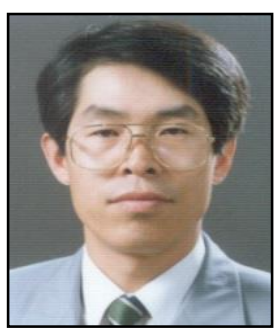

Sung Jin Cho, he received the MS degree and the Ph.D. degree at Korea University. He is currently a professor at Pukyong University since 1988. His research interests include finite field theory, discrete mathematics and cellular automata.

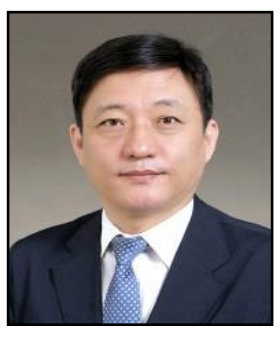

Han Doo Kim, he received the MS degree and the Ph.D. degree at Korea University. He is currently a professor at Inje University since 1989. His research interests include cryptography, cellular automata and its applications.

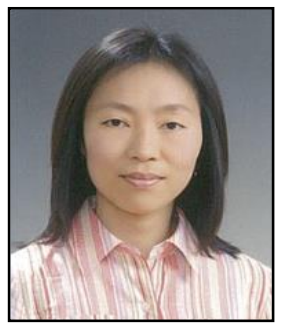

Min Jeong Kwon, she received the MP degree. at Pusan National University and the Ph.D. degree at Pukyong National University. She is currently a teacher of mathematics. Her research interests include finite field theory, discrete mathematics and cellular automata.

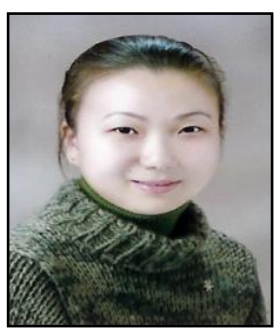

Jin Gyoung Kim, she received the M.S. degree and the Ph.D. degree at Pukyong National University.

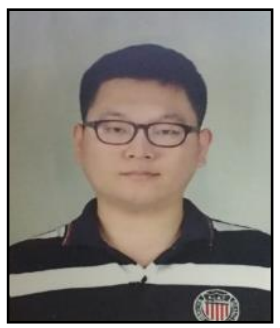

Sung Won Kang, he is currently on the course for the M.S. degree at Pukyong National University. 
International Journal of Control and Automation

Vol. 10, No. 10 (2017) 